\title{
Estudio de foco de paragonimosis en Fuente Clara, Robledo, área periurbana de Medellín, Antioquia
}

\author{
Eudoro Casas¹, Catalina Gómez, 2, Erika Valencia'1,2, Laura Salazar², \\ Luz Elena Velásquez ${ }^{1,2}$ \\ ${ }^{1}$ Grupo Microbiología Ambiental, Escuela de Microbiología, Universidad de Antioquia, Medellín, Colombia \\ 2 Programa de Estudio y Control de Enfermedades Tropicales/PECET, Universidad de Antioquia, Medellín, \\ Colombia
}

Introducción. La información sobre paragonimosis humana en Colombia llevó a suponer que los focos de la enfermedad estaban localizados en sectores selváticos; sin embargo, durante 2005 se hallaron cangrejos infectados con Paragonimus sp. en Fuente Clara, zona urbana de Medellín. Esto motivó la investigación.

Objetivo. Realizar un estudio ecoepidemiológico de la paragonimosis con la participación de la comunidad para promover un manejo adecuado de los ecosistemas acuáticos.

Materiales y métodos. Se realizó búsqueda de huéspedes silvestres y humanos de Paragonimus sp. en Fuente Clara. La presencia de formas larvarias y gusanos adultos del digéneo se evaluó en moluscos, crustáceos y mamíferos. En las personas voluntarias se hizo diagnóstico en esputo. Al agua de la quebrada La Puerta se le midieron: coliformes totales/ fecales, $\mathrm{pH}$, conductividad y oxígeno disuelto. Con niños y adolescentes se realizaron talleres educativos utilizando técnicas lúdicas.

Resultados. Los porcentajes de infección encontrados fueron: caracoles, 0,07\%; cangrejos, $55,5 \%$; mamíferos, $25 \%$, y personas, $0 \%$.

Durante los talleres educativos se manifestó la importancia de los recursos naturales del barrio. Los niños identificaron los huéspedes de Paragonimus sp. y detectaron los factores de riesgo para adquirir la enfermedad.

Se determinó que el agua de la quebrada La Puerta no es apta para el consumo y la recreación de las personas.

Conclusiones. Se señala a Fuente Clara como el primer foco de paragonimosis en zona urbana de Colombia, donde la manipulación y el consumo de cangrejos ponen en riesgo de adquirir la infección a sus habitantes; se sugiere realizar vigilancia de la enfermedad en el sector.

Palabras clave: Paragonimus, tremátodos, zoonosis, epidemiología, educación, salud.

Paragonimosis in the peri-urban zone of Medellín, Antioquia

Introduction. Human paragonimosis in Colombia was assumed to be restricted to the sylvatic areas. However, in 2005, crabs infected with Paragonimus were found in Fuente Clara, an urban sector in Medellín.

Objective. A study was designed to understand the ecoepidemiology of paragonimosis. Programs were initiated to educate the community in the suitable use of wetland ecosystems. Materials and methods. Infection rates of Paragonimus in human and wild hosts was documented in the Fuente Clara sector. The presence of larvae and digenic adult worms was detected in mollusks (1,312), crabs (27) and mammals (4). Sputum diagnosis was performed on samples from 18 volunteer individuals. The following determinants of water quality were measured in the stream "La Puerta": total fecal/coliform, $\mathrm{pH}$, conductivity and dissolved oxygen were measured. Recreational workshops were conducted with children and teenagers for educational purposes.

Results. The percentage of infections found in hosts was as follows: snails, $0.07 \%$; crabs, $55.5 \%$; (wild) mammals, 25\%; humans, 0\%. During the workshops, children and teenagers identified the Paragonimus hosts and the risk factors for acquiring the disease. 
The water of the stream was found to be unsuitable for consumption and recreation (the most probable number of total coliforms $/ 100 \mathrm{ml}$ was in a scale of $10^{4}$ ).

Conclusions. Fuente Clara is the first urban locality in Colombia where a focus of Paragonimus was found. Exposure to and consumption of crabs may constitute a risk for human infection.

Key words: Paragonimus, trematoda, zoonoses, epidemiology, education, health.

Millones de personas padecen de paragonimosis, o distomatosis pulmonar, en diversos países de Asia, África y América; en esta última, el principal agente causal es Paragonimus mexicanus (Trematoda: Digenea). El parásito se hospeda en el pulmón de las personas, y causa síntomas como dolor torácico, tos y hemoptisis, cuadro clínico que por ser similar al de la tuberculosis, puede generar diagnósticos erróneos $(1,2)$.

Esta infección se adquiere al consumir cangrejos de agua dulce, crudos o mal cocidos, que contengan metacercarias del parásito. Aunque el consumo de cangrejos no se supone común, en Colombia esta práctica es habitual bajo diversas condiciones culturales y económicas, debido a su fácil acceso y alto contenido nutricional.

Paragonimus sp. es un endoparásito con un ciclo de vida complejo, que requiere de un molusco como primer huésped intermediario, un crustáceo como segundo y un mamífero como huésped definitivo (3-5). En relación con sus huéspedes, este tremátodo ha mostrado mayor especificidad por los moluscos que, en Suramérica, corresponden a caracoles del género Aroapyrgus (Prosobranchia: Hydrobiidae) $(4,6)$.

En lo que respecta a los crustáceos huéspedes, en América se han registrado diferentes géneros, tales como Ptychophallus, Pseudothelphusa, Potamocarcinus, Strengeria e Hypolobocera $(7,8)$. Asimismo, se han señalado a las personas y a diversos mamíferos silvestres como huéspedes definitivos del digéneo: felinos, mustélidos (Mephitis macrura), cánidos (Canis spp.) y marsupiales; eventualmente, cerdos y roedores (9).

Luz Elena Velásquez, Sede de Investigación Universitaria, Universidad de Antioquia, Calle 62 No. 52-59, laboratorio 730, Medellín, Colombia.

Teléfono: 219 6514; fax: 2196511

luzelena333@yahoo.com

Recibido: 06/03/08; aceptado:28/05/08
}

El único estudio de foco de paragonimosis en Colombia se realizó en un sector rural, donde el $10 \%$ de la población presentó la infección (1). Este estudio sugiere considerar a las personas como reservorios del digéneo cuando, además de consumir cangrejos crudos o mal cocidos, con sus prácticas sanitarias contribuyen a la dispersión de los huevos del parásito.

Registros posteriores de pacientes en el Hospital San Vicente de Paúl de Medellín, procedentes de áreas rurales, llevaron a considerar que los focos de paragonimosis estaban relegados a estos sectores. Sin embargo, durante el primer semestre del 2005 se capturaron cangrejos infectados con metacercarias de Paragonimus sp. en un sector urbano del municipio de Medellín.

Este hallazgo motivó la realización de una investigación ecoepidemiológica en dicho sector, con la participación de la comunidad, para reconstruir con ellos los factores de riesgo a los que se encuentran expuestos, además de incentivarlos a asumir una relación respetuosa con el entorno natural para su beneficio.

\section{Materiales y métodos \\ Área de estudio}

Fuente Clara, ubicada a 6 $16^{\prime} 12^{\prime \prime} \mathrm{N}$ y 75³6'9" O, en el barrio Robledo de Medellín. Presenta una topografía pendiente, rica en fuentes hídricas, entre las que se destacan las quebradas La Iguaná y La Puerta (Plan de Ordenamiento Territorial de Medellín). Ambas son bebederos de animales domésticos y silvestres. En ellas, algunos habitantes de la zona se dedican a la extracción de piedra y arena. La localidad está poblada en gran proporción por familias provenientes de diferentes partes del departamento y del país.

En la quebrada La Puerta los investigadores con la comunidad seleccionaron tres estaciones donde recolectaron moluscos, crustáceos y pequeños mamíferos. Se realizaron muestreos trimestrales, 
con un esfuerzo de captura de cuatro personas durante dos horas, entre los años 2006 y 2007.

Los moluscos se capturaron con redes plásticas (Alba-Tercedor J. Macroinvertebrados acuáticos y calidad de aguas de los ríos. IV Simposio del Agua en Andalucía (SIAGA), Almería, 1996;2:20313) y se transportaron vivos con agua del lugar al Laboratorio de Malacología Médica y Tremátodos del Programa de Estudio y Control de Enfermedades Tropicales (PECET), donde se instalaron en acuarios adecuados con agua declorada y aireación artificial, a la temperatura y $\mathrm{pH}$ del hábitat natural. Se alimentaron con lechuga fresca. Algunos ejemplares se sacrificaron para su determinación taxonómica (10).

Luego de 48 horas de aclimatación, se seleccionaron los moluscos identificados como Aroapyrgus, que se individualizaron en cajas de ELISA de 24 pozos, con agua declorada, para estimular la emisión de cercarias, bajo una fuente luminosa por seis horas. Se revisaron cada tres horas al estereomicroscopio en busca de cercarias de Paragonimus sp. Este procedimiento se realizó cada 15 días con los caracoles no emisores y se replicó en seis oportunidades, dando espacio a la maduración de las larvas del tremátodo (comunicación personal, Mercedes Villa Lloberas, Universidad de Barcelona). Los ejemplares de Aroapyrgus no emisores se relajaron con nembutal al $0,05 \%$, se sacrificaron y se disectaron para buscar larvas del digéneo, que se identificaron según sus caracteres morfológicos. Se calculó el porcentaje de infección en los moluscos.

Los cangrejos se recolectaron con la mano, se llevaron vivos al laboratorio con agua del lugar. Se sacrificaron con choque térmico, sumergiéndolos en agua a $2^{\circ} \mathrm{C}$ durante 10 minutos. Se disecaron y examinaron al estereomicroscopio en búsqueda de metacercarias, las cuales se fotografiaron y midieron para identificarlas según sus caracteres morfológicos.

Se determinó el estatus taxonómico de los crustáceos por la morfología de los gonópodos y del tercer maxilípedo $(11,12)$. Se hicieron registros fotográficos y se depositaron ejemplares en la colección de crustáceos del Museo Universitario
(Universidad de Antioquia). Se estimó el porcentaje de infección en los cangrejos.

Los mamíferos se capturaron con trampas Tomahawk® con cebo con plátano maduro y mantequilla de maní, instaladas entre la vegetación que bordea la quebrada La Puerta y en algunas viviendas del sector. Se les tomó una muestra de materia fecal para hacer diagnóstico parasitológico de Paragonimus sp. por la técnica de Dennis, modificada y estandarizada en el Laboratorio de Malacología Médica y Tremátodos del PECET. Se observaron huevos al microscopio; se hicieron registros fotográficos.

Los mamíferos se sacrificaron para determinar la presencia del parásito adulto en los pulmones. Se anestesiaron utilizando una combinación de acepromacina, $0,1 \mathrm{mg} / \mathrm{kg}$, y ketamina, $10 \mathrm{mg} / \mathrm{kg}$, por vía intramuscular, hasta el estado de anestesia profunda. Se sometieron a la eutanasia con Euthanex ${ }^{\circledR} 1 \mathrm{ml} / \mathrm{kg}$ por vía intracardiaca (13). Se calculó el porcentaje de infección.

Los ejemplares adultos de Paragonimus sp. se pusieron en solución salina a temperatura ambiente para estimular la ovipostura. Los huevos se lavaron con agua destilada y se almacenaron a $4{ }^{\circ} \mathrm{C}$. Algunos adultos se fijaron para estudios morfológicos en una solución de alcohol al 95\%, formol al 37\%, ácido acético y agua destilada, y los demás se preservaron en alcohol al $75 \%$ a $20{ }^{\circ} \mathrm{C}$, para futuros estudios en genética molecular.

Durante las salidas de campo en la quebrada La Puerta se hicieron análisis físico-químico y microbiológico. Se estimaron el pH con un medidor digital $\mathrm{PH}-2000$, la conductividad con un conductivímetro y el oxígeno disuelto en el agua por el método de Winkler (14). También se calculó el número más probable de coliformes totales y fecales por el método de tubos múltiples de fermentación (15).

Se hizo un muestreo por conveniencia de tosedores crónicos en las zonas aledañas a la quebrada La Puerta, tomando una muestra voluntaria de esputo; los donantes recibieron las indicaciones necesarias para la correcta obtención de la muestra. 
Las muestras de esputo se trataron con formalina al $10 \%$ y se expusieron a luz ultravioleta durante 30 minutos; después se lavaron con agua destilada. Se vaciaron a cajas de Petri y se buscaron los huevos de Paragonimus sp. al estereomicroscopio (16).

Se realizaron talleres educativos lúdicos con la comunidad y algunas instituciones educativas de Fuente Clara en los que se invitó a niños y adolescentes a manifestar por medio del dibujo su apreciación sobre los recursos naturales de su barrio. A partir de estos dibujos, se reconstruyó el ciclo de vida de Paragonimus sp. y se identificaron los factores de riesgo para adquirir la infección.

En estos talleres se estimularon las reflexiones sobre la paragonimosis en la búsqueda de estrategias para la prevención de la enfermedad, utilizando como referente el tipo de interacción que tienen los habitantes del sector con las fuentes de agua del vecindario.

Para afianzar la relación de la comunidad con la institución académica, se realizó una visita guiada hasta las instalaciones del Laboratorio de
Malacología Médica y Tremátodos del PECET, con 42 personas de Fuente Clara. Durante la visita conocieron los elementos del ciclo de vida de Paragonimus sp. obtenidos en su localidad y las herramientas y equipos de uso frecuente en el laboratorio para los estudios de tremátodos. El personal universitario se ocupó de instruir y explicar las inquietudes de estas personas.

\section{Aspectos éticos}

El protocolo del presente trabajo cumplió los parámetros establecidos por el Área Metropolitana del Valle de Aburrá para investigación científica en diversidad biológica, según la Resolución № 000630 del 20 de septiembre de 2006; además, contó con el consentimiento verbal de la Junta de Acción Comunal del sector de Fuente Clara, en representación de la comunidad, para la realización de las actividades programadas en el estudio. A quienes donaron voluntariamente muestras de esputo se les solicitó la firma de consentimiento informado.

\section{Resultados}

Los caracoles identificados como Aroapyrgus colombiensis (figura 1A) (4) emitieron cercarias

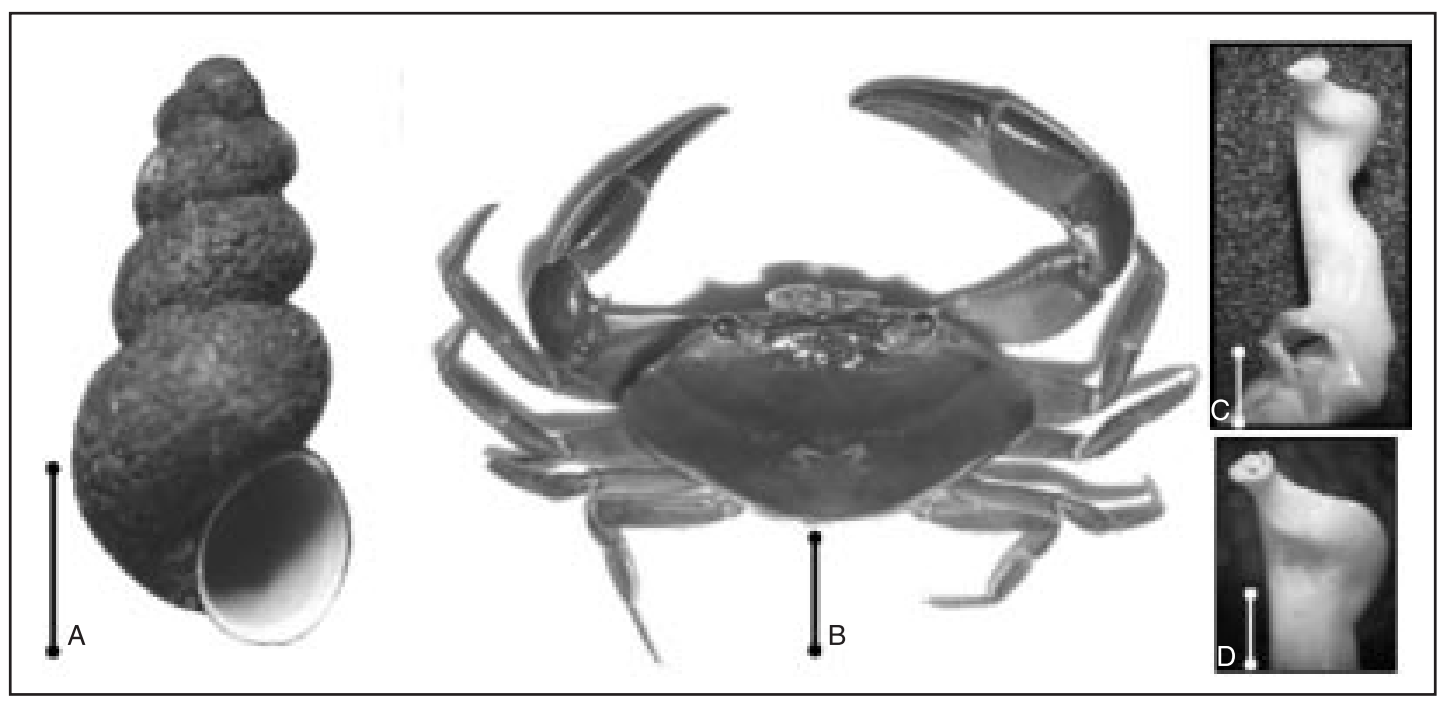

Figura 1. Huéspedes intermediarios de Paragonimus sp. capturados en la quebrada La Puerta. A: Aroapyrgus colombiensis; barra: $1 \mathrm{~mm}$. B: Hypolobocera bouvieri; barra: $1 \mathrm{~cm}$. C: gonópodo izquierdo de Hypolobocera bouvieri; barra: $2 \mathrm{~mm}$. D: detalle de la región cefálica del gonópodo izquierdo; barra: $1 \mathrm{~mm}$. Fotografías tomadas en el Laboratorio de Malacología Médica y Tremátodos. 
Cuadro 1. Frecuencia de infección de Paragonimus sp. y de Heterophyidae en los moluscos Aroapyrgus colombiensis recolectados en la quebrada La Puerta, Fuente Clara, Medellín.

\begin{tabular}{|c|c|c|c|}
\hline Salida de campo & $\begin{array}{l}\text { A. colombiensis } \\
\text { colectados }\end{array}$ & $\begin{array}{l}\text { A. colombiensis } \\
\text { con cercarias de } \\
\text { Paragonimus }\end{array}$ & $\begin{array}{l}\text { A. colombiensis } \\
\text { con cercarias de } \\
\text { un Heterophyidae }\end{array}$ \\
\hline Primera & 12 & $0(0 \%)$ & $6 \quad(50,00 \%)$ \\
\hline Segunda & 195 & $0(0 \%)$ & $42(21,53 \%)$ \\
\hline Tercera & 203 & $0(0 \%)$ & 10 (4,90 \%) \\
\hline Cuarta & 902 & $1(0,11 \%)$ & $0 \quad(0 \%)$ \\
\hline Total & 1.312 & $1(0,07 \%)$ & $58(4,42 \%)$ \\
\hline
\end{tabular}

que presentaron la morfología típica de Paragonimus sp.: ventosa oral armada con un estilete, rodeada de canalículos conectados a las glándulas de penetración, ubicadas alrededor del acetábulo; cola pequeña, redondeada y con espinas (5). La frecuencia de infección en los moluscos fue baja $0,07 \%$ (cuadro 1 ).

Durante la evaluación de los moluscos se presentó el hallazgo incidental de otro digéneo, el cual se asignó a la familia Heterophyidae, cuyas cercarias son más pequeñas, presentan dos manchas oculares, ventosa oral sin estilete y cola dos veces más larga que el cuerpo; su frecuencia fue mayor que la de Paragonimus (cuadro 1).

Se capturaron 27 cangrejos de la familia Pseudothelphusidae, determinados como Hypolobocera bouvieri (figura 1B). En $55,5 \%$ de éstos se encontraron metacercarias de Paragonimus sp., con un promedio de seis por espécimen (cuadro 2). Presentaban cuerpo color rosa, ventosa oral más pequeña que el acetábulo, seguida de una faringe corta, que se bifurca en dos ciegos intestinales sinuosos, laterales y proyectados hasta el extremo posterior, acetábulo ecuatorial y sistema excretor negro entre los ciegos. Las metacercarias midieron $0,84 \mathrm{~mm}$ a $1,18 \mathrm{~mm}$ de largo $\times 0,46 \mathrm{~mm}$ a $0,54 \mathrm{~mm}$ de ancho (figura 2A).

Se capturaron cuatro mamíferos, un Ratus sp. (Rodentia) y tres Didelphis marsupialis (Marsupialia). En las heces de un marsupial se encontraron huevos con la morfología de Paragonimus sp. De los pulmones de este mamífero se obtuvieron nueve adultos del parásito (cuadro 3), distribuidos en cuatro quistes, de 10 a $12 \mathrm{~mm}$ de largo.
Cuadro 2. Frecuencia de infección por metacercarias de Paragonimus sp. en Hypolobocera bouvieri capturados en la quebrada La Puerta, Fuente Clara, Medellín.

\begin{tabular}{lcc}
\hline Salida de campo & $\begin{array}{c}\boldsymbol{H} \text {. bouvieri } \\
\text { capturados }\end{array}$ & $\begin{array}{c}\boldsymbol{H} \text {. bouvieri con } \\
\text { metacercarias } \\
\text { de Paragonimus }\end{array}$ \\
\hline Primera & 20 & $8(40 \%)$ \\
Segunda & 4 & $4(100 \%)$ \\
Tercera & 2 & $2(100 \%)$ \\
Cuarta & 1 & $1(100 \%)$ \\
Total & $\mathbf{2 7}$ & $\mathbf{1 5}(\mathbf{5 5 , 5 \% )}$ \\
\hline
\end{tabular}

Los adultos de Paragonimus sp. eran ovalados y de color rosa. En fresco midieron de $9 \mathrm{~mm}$ a 11 $\mathrm{mm}$ de largo $\times 4 \mathrm{~mm}$ a $5 \mathrm{~mm}$ de ancho; el acetábulo varió de $0,7 \mathrm{~mm}$ a $0,9 \mathrm{~mm}$ de largo $\times 0,8 \mathrm{~mm}$ a $0,9 \mathrm{~mm}$ de ancho. La ventosa oral redonda midió $0,7 \mathrm{~mm}$ a $1,0 \mathrm{~mm}$ de diámetro (figura 2B).

La morfología de los huevos de Paragonimus sp. obtenidos de los parásitos adultos y de las heces del didélfido fue idéntica: coloración amarillo ocre, ovalados, de pared gruesa y con opérculo en un extremo. Los huevos midieron entre $70 \mu \mathrm{m}$ y 81 $\mu \mathrm{m}$ (media, 65,6) de largo x $50 \mu \mathrm{m}$ a $60 \mu \mathrm{m}$ (media, 52,7 ) de ancho (figura $2 \mathrm{C}$ ).

Se recogieron 18 muestras de esputo de personas tosedoras crónicas de Fuente Clara, 10 en mujeres y 8 en hombres, todas con diagnóstico negativo para huevos de Paragonimus sp.

En el análisis microbiológico del agua de la quebrada La Puerta se encontró una tendencia al incremento del número más probable de coliformes totales y fecales en el sentido de la corriente (cuadro 4), en tanto que las variables 


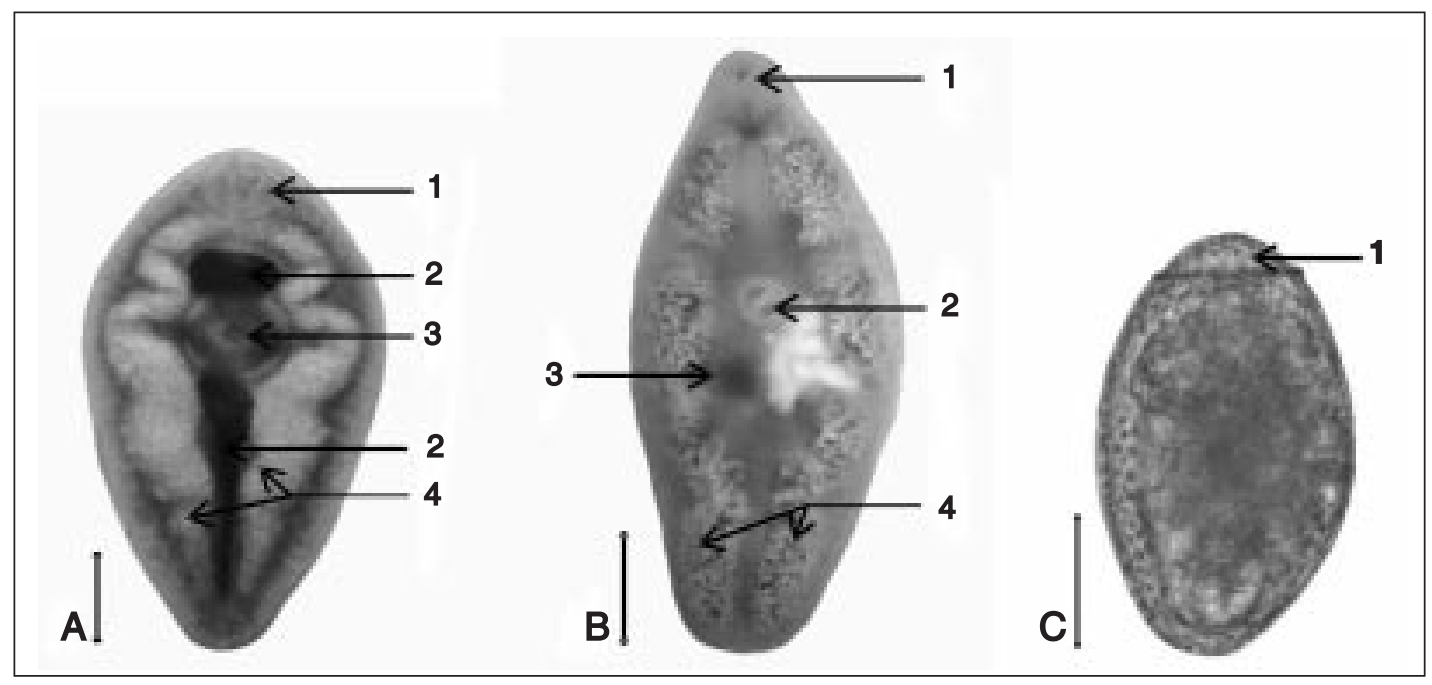

Figura 2. Paragonimus mexicanus capturado en Fuente Clara. A: Metacercaria. 1. Ventosa oral, 2. Sistema excretor, 3. Acetábulo, 4. Ciegos intestinales. Barra: 0,2 mm. B: Adulto. 1. Ventosa oral, 2. Acetábulo, 3. Ovario, 4. Conductos vitelarios. Barra: 2 mm. C: Huevo. 1. Opérculo. Barra: $25 \mu \mathrm{m}$. Fotografías tomadas en el Laboratorio de Malacología Médica y Tremátodos.

Cuadro 3. Frecuencia de infección por Paragonimus sp. en mamíferos capturados en Fuente Clara, Medellín.

\begin{tabular}{lcc}
\hline Salida de campo & $\begin{array}{c}\text { Mamíferos } \\
\text { capturados }\end{array}$ & $\begin{array}{c}\text { Mamíferos } \\
\text { infectados } \\
\text { con Paragonimus }\end{array}$ \\
\hline Primera & 3 & $1(33,33 \%)$ \\
Segunda & 0 & $0(0 \%)$ \\
Tercera & 1 & $0(0 \%)$ \\
Cuarta & 0 & $0(0 \%)$ \\
Total & $\mathbf{4}$ & $\mathbf{1}(\mathbf{2 5 \% )}$ \\
\hline
\end{tabular}

Cuadro 4. Análisis microbiológico del agua de la quebrada La Puerta por estación de muestreo.

\begin{tabular}{lcc}
\hline № estación & $\begin{array}{c}\text { Número más } \\
\text { probable de } \\
\text { coliformes } \\
\text { totales/100 } \mathbf{~ m l}\end{array}$ & $\begin{array}{c}\text { Número más } \\
\text { probable de } \\
\text { coliformes } \\
\text { fecales } / \mathbf{1 0 0} \mathbf{~ m l}\end{array}$ \\
\hline 1 & $21-26 \times 10^{4}$ & $14-21 \times 10^{4}$ \\
2 & $60-70 \times 10^{4}$ & $17-26 \times 10^{4}$ \\
3 & $80-90 \times 10^{5}$ & $14-23 \times 10^{5}$ \\
\hline
\end{tabular}

físico-químicas estimadas en el agua no mostraron mayores diferencias (cuadro 5).

En las prácticas de campo realizadas con los niños de la comunidad, se observó la pericia de la mayoría para manipular los cangrejos, llegando algunos a mantenerlos como mascotas. Además,
Cuadro 5. Análisis físico-químico del agua de la quebrada La Puerta por estación de muestreo.

\begin{tabular}{lccc}
\hline $\begin{array}{l}\text { No } \\
\text { estación }\end{array}$ & pH & $\begin{array}{c}\text { Oxígeno } \\
\text { disuelto } \\
\text { (ppm) }\end{array}$ & $\begin{array}{c}\text { Conductividad } \\
\boldsymbol{\mu S} / \mathbf{c m}\end{array}$ \\
\hline 1 & $7,3-7,8$ & $7,1-7,6$ & $101-156$ \\
2 & $7,2-7,9$ & $6,9-7,9$ & $95-153$ \\
3 & $7,6-7,8$ & $6,8-7,6$ & $99-160$ \\
\hline
\end{tabular}

de ellos se obtuvo información sobre el consumo de dichos crustáceos por parte de varias familias.

En los talleres educativos se percibió que los niños y los adolescentes de Fuente Clara valoran los recursos naturales de su barrio como una gran riqueza, según lo demuestran las ilustraciones que realizaron sobre los animales, las plantas y la quebrada La Puerta. Durante estas actividades se logró, además, que relacionaran a los cangrejos, los caracoles y las zarigüeyas con el ciclo de vida de Paragonimus sp. y que reconocieran la manipulación de los crustáceos y su consumo, crudos o semicocidos, como un factor de riesgo para adquirir la paragonimosis.

Con la visita al Laboratorio de Malacología Médica y Tremátodos del PECET de los representantes de Fuente Clara, se afianzó el conocimiento 
adquirido sobre paragonimosis, se incrementó la confianza con la institución universitaria y se estrecharon los nexos entre ambos, lo cual dio lugar a nuevas propuestas de investigación en el barrio.

\section{Discusión}

El hallazgo de todos los huéspedes infectados con Paragonimus sp. en Fuente Clara permite señalarla como el primer foco descrito de paragonimosis en una zona urbana de Colombia. Este hecho revalúa el supuesto de que los focos de la infección estaban limitados a los sectores selváticos $y$, por ende, alejados de las áreas urbanas $(1,17)$.

Este constituye el primer registro de larvas de un Heterophyidae en Aroapyrgus colombiensis que, además, se encuentra en simpatría con Paragonimus sp. La diferencia en las frecuencias de infección encontradas entre ambos parásitos lleva a considerar la necesidad de establecer el posible impacto de un parásito sobre la dinámica de la población del otro (18).

La baja frecuencia de Paragonimus sp. en los caracoles contrasta con la alta frecuencia en los crustáceos, lo que demuestra la eficiencia de $A$. colombiensis como vector del parásito. Se han registrado resultados similares en otros países latinoamericanos $(7,19)$.

La facilidad para diagnosticar Paragonimus sp. en los cangrejos, el constituir la fuente de infección para los mamíferos y la alta frecuencia del parásito en ellos, permiten proponerlos como indicadores de focos de paragonimosis y como el agente principal en las campañas de prevención de la enfermedad.

El ejemplar de Didelphis marsupialis infectado con Paragonimus mexicanus constituye el primer reservorio silvestre registrado en un foco de paragonimosis en Antioquia. En el foco de Valle de Pérdidas no se estableció el reservorio silvestre; en cambio se sugirió el desempeño de la población humana como reservorio del digéneo (1).

Aunque los pacientes sintomáticos respiratorios de Fuente Clara no se encontraron infectados con Paragonimus sp., se recomienda diseñar un programa de vigilancia de la enfermedad, debido a la frecuente manipulación de los cangrejos, que pone en riesgo a los habitantes del sector de infectarse con el parásito.

La quebrada La Puerta permanece con caudal durante todo el año y se mantiene oxigenada debido a la pendiente y el lecho rocoso (Villarroya GF. La gestión de las aguas superficiales y el medio ambiente. Seminario Permanente sobre Ciencias de la Tierra y del Medio Ambiente. 12 de diciembre de 2000. Madrid: Universidad Complutense de Madrid; 2001). Sin embargo, el número más probable de coliformes totales y fecales indica que no es apta para el consumo humano, ni para la recreación, según los valores permitidos en el Decreto 475 de 1998 del Ministerio de Salud de Colombia.

Ante el interés de los niños y de los adolescentes de Fuente Clara por sus recursos naturales, se sugiere fomentar una buena relación entre la comunidad y estos recursos, desde las instituciones educativas, con el fin de mejorarlos y hacerlos aptos para la recreación.

Para explicarle a la comunidad el riesgo de la infección con Paragonimus sp., es necesario ayudarla a identificar y comprender los elementos de su vida cotidiana que la expone al parásito. Este propósito se alcanza con su participación en las propuestas desde el inicio de las mismas.

\section{Agradecimientos}

A la Junta de Acción Comunal y a la comunidad de Fuente Clara, por su participación permanente en la realización del trabajo, a la bacterióloga Auxilio Ramírez por su asesoría. A Iván Darío Vélez, director del PECET, por su apoyo a las investigaciones sobre trematodiasis, y al personal del Laboratorio de Malacología Médica y Tremátodos por su acompañamiento.

\section{Conflictos de intereses}

Los autores declaramos que no existen conflictos de intereses en el desarrollo y publicación de este trabajo.

\section{Financiación}

Esta investigación fue financiada por el Comité para el Desarrollo de la Investigación (CODI) de la Universidad de Antioquia. 


\section{Referencias}

1. Vélez ID, Velásquez LE. Paragonimosis: una investigación multidisciplinaria en salud, biología y cultura en Colombia. Medellín: Universidad de Antioquia; 2002. p. 7-8.

2. Churg $\mathbf{C H}$. Human paragonimiasis. Pathology of protozoal and helminthic diseases with clinical correlation. Baltimore: Williams and Wilkins; 1971. p. 504.

3. Blair D, Xu ZB, Agatsuma T. Paragonimiasis and the genus Paragonimus. Adv Parasitol. 1999;42:113222.

4. Malek E, Little MD. Aroapyrgus colombiensis n. sp. (Gastropoda: Hydrobiidae), snail intermediate host of Paragonimus caliensis in Colombia. The Nautilus. 1971;85:20-6.

5. Vélez I, Velásquez LE, Vélez ID. Morphological description and life cycle of Paragonimus sp. (Trematoda: Troglotrematidae): causal agent of human paragonimiasis in Colombia. J. Parasitol. 2003;89:74955.

6. Lamothe-Argumedo R, Malek EA, MeaveGallegos 0 . Aroapyrgus alleei Morrison, (Gastropoda: Hydrobiidae) first intermediate host of Paragonimus mexicanus in Colima, Mexico. J Parasitol. 1983;69:226-8.

7. Lamothe-Argumedo R, Caballero J, Lázaro E. Pseudothelphusa $(P)$. dilatata Rathbun (Crustacea: Decapoda), segundo hospedador intermediario de Paragonimus mexicanus (Trematoda). An Inst Biol Unif Nal Auton México. 1976;48:295-8.

8. Lamothe-Argumedo R. La paragonimiasis en el continente americano. Salud Pública Mex. 1985; 27 : 514-23.

9. Caballero YC, Montero-Gei F. Descripción de dos tremátodos de un marsupial de la República de Costa Rica y un catálogo de los tremátodos que parasitan a Marsupialia illger, 1811. Ann Esc Nac Cien Biol. (México) 1961;10:45-86.

10. Moné $\mathbf{H}$, Théron $\mathbf{A}$, Combes $\mathbf{C}$. Interaction between the Biomphalaria glabrata-Schistosoma mansoni hostparasite system and the non-target mollusks: influence on cercarial production. J. Parasitol. 1986;72:410-6.

11. Campos MR, Rodriguez G. Two new species of freshwater crabs of the genus Hypolobocera from Colombia (Crustacea: Decapoda: Pseudothelpusidae). Proc Biol Soc Wash. 1995;108:649-55.

12. Campos MR. Freshwater crabs from Colombia a taxonomic and distributional study. Bogotá, D.C: Editora Guadalupe Ltda.; 2005. p. 224-94.

13. Vélez A. Guías de parasitología veterinaria. Medellín: Editorial Exitodinámica; 1983. p. 306.

14. Fuentes F, Masol-Deya A. Manual de Laboratorio Ecología de los microorganismos. Pt. 3. Puerto Rico: Universidad de Puerto Rico; 2002. p. 1-15.

15. The American Public Health Association, The American Water Works Association, Water Pollution Control Federation. Standard methods for the examination of water and wastewater. $15^{\text {th }}$ ed. Washington, D.C. American Public Health Association; 1980.

16. Vélez I, Ortega J, Velásquez LE. Paragonimiasis: a view from Colombia. Clin Chest Med. 2002;23:421-31.

17. Little MD. Paragonimus caliensis sp. and paragonimiasis in Colombia. J Parasitol. 1968;54:738-46.

18. Velásquez LE, Bedoya JC, Areiza A, Vélez I. Primer registro de Centrocestus formosanus (Digenea: Heterophyidae) en Colombia. Revista Mexicana de Biodiversidad. 2006;77:119-21.

19. Velásquez LE, Restrepo S, Gómez MI, Vélez ID. Aspectos ecoepidemiológicos del caracol Aroapyrgus sp. Revista Asociación Colombiana de Ciencias Biológicas. 1999;11:7-15. 